\title{
STOCHASTIC FLUTTER OF MULTI-STABLE NON-LINEAR AIRFOIL IN TURBULENT FLOW
}

\section{YING HAO}

School of Mechanical Engineering, Tianjin University, Tianjin, China, and

College of Civil Engineering and Mechanics, Yanshan University, Qinhuangdao, China

e-mail: haoying@tju.edu.cn

\section{ZHI-QIANG WU}

School of Mechanical Engineering, Tianjin University, Tianjin, China

\begin{abstract}
Stochastic airfoil flutter in an unsteady flow is discussed using the stochastic P-bifurcation method, taking into account potential effects of the longitudinal and vertical turbulent flow. The critical conditions of stochastic P-bifurcation are deduced by stochastic singularity analysis in order to discuss stochastic P-bifurcation phenomena. The results of parameter analysis show that as the turbulent intensity increases, the critical flutter velocity for sharp stochastic airfoil flutter decreases. And the large amplitude vibration comes earlier; an increase in the turbulent scale causes an earlier appearance of the critical velocity for large amplitude stochastic flutter.
\end{abstract}

Keywords: stochastic P-bifurcation, turbulence, stochastic flutter

\section{Nomenclature}

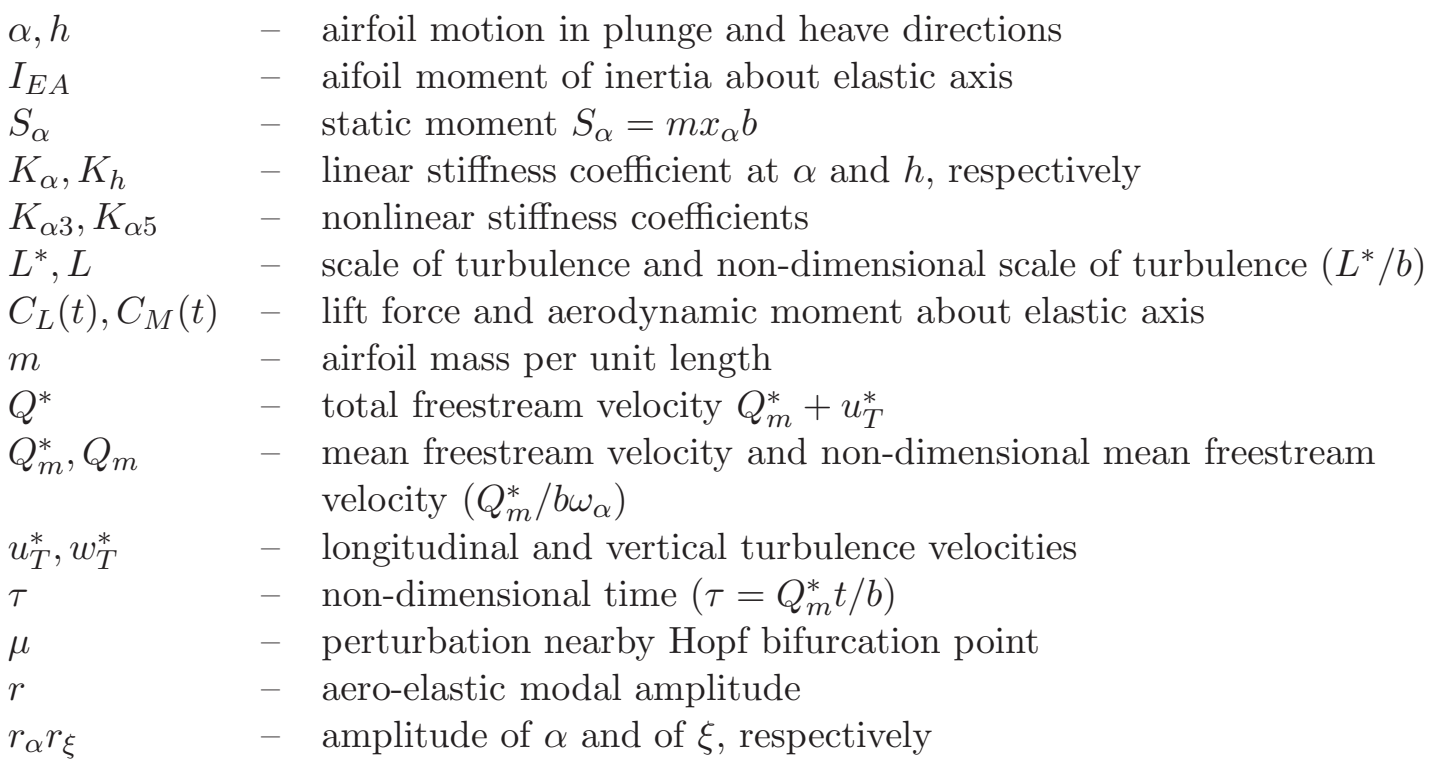

\section{Introduction}

Turbulence and other stochastic perturbation sources are often ignored in airfoil flutter studies, though turbulence-induced stochastic perturbation does exist during aircraft flight. Atmospheric turbulence, for example, plays a great role in aircraft design. 
In the 1990s, Poirel and Price (1997, 2001, 2003b, 2007), Poirel et al. (2005, 2006) and his colleagues pioneered researches on turbulence-induced stochastic flutter by addressing the existence of stochastic turbulent perturbation in their studies. Poirel and Price (1997) examined stability of an airfoil model with structural nonlinearity in the pitch direction by incorporating the effects of turbulence into the longitudinal inflow velocity presented in Price et al. (2013). In Lee et al. (1999), a fairly complete description of airfoil flutter with structural or aerodynamic nonlinearity was involved. Numerically investigated were the effects of longitudinal turbulent perturbation on the system Hopf bifurcation and airfoil flutter boundary. Poirel and Price (2003a) addressed the effects of both longitudinal and vertical turbulences in their airfoil model. Poirel and Price (2007) pioneered a stochastic bifurcation study of the airfoil flutter system by looking at the effects of stochastic P-bifurcation of a binary airfoil model respecting to turbulent intensity and system nonlinear rigidity through Monte-Carlo simulation. It was found that the bifurcation was characterized by a change in shape of the response probability structure, while no discontinuity in the variation of the largest Lyapunov exponent when airspeed was observed.

Zheng (2007) analyzed the airfoil RMS(root mean square) response of a binary airfoil model of a deterministic system with double stable limit cycle flutter using stochastic theory taking into account the effects of flow velocity and turbulent intensity on the system RMS. Zhao (2009) investigated stochastic flutter of a binary airfoil model of a deterministic system with Hopf bifurcation, and described the system stochastic bifurcation with the system joint probability density, marginal probability density and the maximum Lyapunov exponent. Huang et al. (2010) analyzed stochastic flutter of an airfoil model with Hopf bifurcation, and discussed the stochastic stability of the airfoil under parametrically-induced white Gaussian noise through Monte Carlo numerical simulation. Chassaing et al. (2012) looked at system stochastic limit cycle oscillation (LCO) by applying an adaptive spectrogram to stochastic nonlinear aerodynamic modeling.

Some scholars have also carried out experimental research on the airfoil flutter. Poirel et al. conducted a number of wind tunnel tests on NACA0012 airfoil. Poirel and Yuan (2010) and Poirel et al. (2008) examined how LCO took place under low Reynolds numbers, and how LCO in the airfoil pitch direction took place in response to laminar separation. Yuan et al. (2013) and Poirel and Mendes (2014) observed mild and sharp LCO under different initial conditions.

Current research of the airfoil flutter system under stochastic perturbation is not all-inclusive yet; few authors have turned their eyes to stochastic turbulent perturbation and airfoil multi-stability. Nevertheless, studying stochastic flutter of an aircraft under turbulent perturbation is of great theoretical and referential importance to airfoil design. The complexity and particularity of the aero-elastic system have confined theoretical solutions for stochastic airfoil flutter to low-dimensional simple systems, which merely address simple system bifurcation such as Hopf bifurcation and rarely involve complex or stochastic bifurcation. Also, studies of high-dimensional, nonlinear stochastic flutter are mostly limited to numerical or experimental approaches. Hence, it becomes extremely necessary to develop an efficient, accurate, highly applicable theoretical method to examine the effects of stochastic turbulence and other parameters.

This paper discusses stochastic airfoil flutter in a turbulence flow through stochastic P-bifurcation analysis, taking into account the potential effects of longitudinal and vertical turbulent intensity and scale. The modified averaging method is applied herein to reduce the system dimension. The critical parametric conditions for stochastic P-bifurcation of the airfoil are deduced by means of stochastic averaging and singularity theory.

\section{High-dimensional airfoil flutter mathematical model}

Restricting the flow to incompressible conditions, but retaining unsteady (lag) effects, the basic structural equations are obtained from (Poirel and Price, 2007) and modified to account for a 
non-linear hardening quantic torsional stiffness, this is the source of nonlinearity considered in this paper

$$
\begin{aligned}
& m \ddot{h}+S_{\alpha} \ddot{\alpha}+D_{h} \dot{h}+K_{h} h+G(h)=-C_{L}(t) \\
& S_{\alpha} \ddot{h}+I_{E A} \ddot{\alpha}+D_{\alpha} \dot{\alpha}+K_{\alpha} \alpha+M(\alpha)=C_{M}(t)
\end{aligned}
$$

$C_{L}(t)$ and $C_{M}(t)$ represent, respectively, the aerodynamic lift force and the aerodynamic moment about the elastic axis. Our study only considered the structural nonlinear rigidity in the pitch direction, $M(\alpha)=K_{\alpha 3} \alpha^{3}+K_{\alpha 5} \alpha^{5}$, and ignored in the plunge direction. Hence $G(h)=0$ has been selected. The aerodynamic lift and aerodynamic moment are expressed as

$$
\begin{aligned}
& C_{L}(t)=-\pi \rho b^{2}\left(\ddot{h}-Q^{*} \dot{\alpha}-b a_{h} \ddot{\alpha}\right)-2 \pi \rho b Q^{*}\left(W_{3 / 4}^{*} \varphi(0)-\int_{0}^{t} W_{3 / 4}^{*}(s) \frac{d \varphi(t-s)}{d s} d s\right) \\
& -2 \pi \rho b Q_{m}^{*}\left(w_{T}^{*} \psi(0)-\int_{0}^{t} w_{T}^{*}(s) \frac{d \psi(t-s)}{d s} d s\right) \\
& C_{M}(t)=\pi \rho b^{2}\left[b a_{h} \ddot{h}-b\left(\frac{1}{2}-a_{h}\right) Q^{*} \dot{\alpha}-b^{2}\left(a_{h}^{2}+\frac{1}{8}\right) \ddot{\alpha}\right]+2 \pi \rho b^{2}\left(a_{h}+\frac{1}{2}\right) Q^{*}\left(W_{3 / 4}^{*} \varphi(0)\right. \\
& \left.-\int_{0}^{t} W_{3 / 4}^{*}(s) \frac{d \varphi(t-s)}{d s} d s\right)+2 \pi \rho b^{2}\left(a_{h}+\frac{1}{2}\right) Q_{m}^{*}\left(w_{T}^{*} \psi(0)-\int_{0}^{t} w_{T}^{*}(s) \frac{d \psi(t-s)}{d s} d s\right)
\end{aligned}
$$

where: $W_{3 / 4}^{*}=\dot{h}+Q^{*} \alpha+b\left(0.5-a_{h}\right) \dot{\alpha}, Q^{*}=Q_{m}^{*}+u_{T}^{*}$, and these terms are influenced by the turbulent excitation, which is composed of a constant mean part $Q_{m}^{*}$ and a time-varying component $u_{T}^{*}$ created by the longitudinal turbulence excitation. In Eq. $(2.2)_{2}$, the unsteady effects are accounted for using the representation of the function $\varphi(t)$, and Küssner's function $\psi(t)$ (Poirel and Price, 2007)

$$
\begin{array}{lccc}
\varphi(t)=1-A_{1} \exp \left(\frac{-b_{1} Q_{m}^{*}}{b} t\right)-A_{2} \exp \left(\frac{-b_{2} Q_{m}^{*}}{b} t\right) & \\
\psi(t)=1-A_{3} \exp \left(\frac{-b_{3} Q_{m}^{*}}{b} t\right)-A_{4} \exp \left(\frac{-b_{4} Q_{m}^{*}}{b} t\right) & \\
A_{1}=0.165 & A_{2}=0.335 & b_{1}=0.115 & b_{2}=0.3 \\
A_{3}=0.5792 & A_{4}=0.4208 & b_{3}=0.1393 & b_{4}=1.802
\end{array}
$$

For the purpose of analysis and calculation, the unsteady part of aerodynamic models $C_{L}(t)$ and $C_{M}(t)$ is usually expressed as a solution to a differential equation. And two new variables $R_{1}^{*}$ and $R_{2}^{*}$ are introduced, then $C_{L}(t)$ and $C_{M}(t)$ can be expressed as

$$
\begin{aligned}
& C_{L}(t)=-\pi \rho b^{2}\left(\ddot{h}-Q^{*} \dot{\alpha}-b a_{h} \ddot{\alpha}\right)-2 \pi \rho b Q^{*}\left[W_{3 / 4}^{*} \varphi(0)+\dot{R}_{1}^{*}\left(A_{1} \bar{b}_{1}+A_{2} \bar{b}_{2}\right)\right. \\
& \left.\quad+R_{1}^{*} \bar{b}_{1} \bar{b}_{2}\left(A_{1}+A_{2}\right)\right]-2 \pi \rho b Q_{m}^{*}\left[w_{T}^{*} \psi(0)+\dot{R}_{2}^{*}\left(A_{3} \bar{b}_{3}+A_{4} \bar{b}_{4}\right)+R_{2}^{*} \bar{b}_{3} \bar{b}_{4}\left(A_{3}+A_{4}\right)\right] \\
& C_{M}(t)=\pi \rho b^{2}\left[b a_{h} \ddot{h}-b\left(\frac{1}{2}-a_{h}\right) Q^{*} \dot{\alpha}-b^{2}\left(a_{h}^{2}+\frac{1}{8}\right) \ddot{\alpha}\right] \\
& \quad+2 \pi \rho b^{2}\left(a_{h}+\frac{1}{2}\right) Q^{*}\left[W_{3 / 4}^{*} \varphi(0)+\dot{R}_{1}^{*}\left(A_{1} \bar{b}_{1}+A_{2} \bar{b}_{2}\right)+R_{1}^{*} \bar{b}_{1} \bar{b}_{2}\left(A_{1}+A_{2}\right)\right] \\
& \quad+2 \pi \rho b^{2}\left(a_{h}+\frac{1}{2}\right) Q_{m}^{*}\left[w_{T}^{*} \psi(0)+\dot{R}_{2}^{*}\left(A_{3} \bar{b}_{3}+A_{4} \bar{b}_{4}\right)+R_{2}^{*} \bar{b}_{3} \bar{b}_{4}\left(A_{3}+A_{4}\right)\right]
\end{aligned}
$$

where

$$
\bar{b}_{1}=\frac{b_{1} Q_{m}^{*}}{b} \quad \bar{b}_{2}=\frac{b_{2} Q_{m}^{*}}{b} \quad \bar{b}_{3}=\frac{b_{3} Q_{m}^{*}}{b} \quad \bar{b}_{4}=\frac{b_{4} Q_{m}^{*}}{b}
$$


Some additional details of the turbulence modelling are provided by Poirel and Price (Poirel and Price, 2003a, 2007; Price et al., 2013; Lee et al., 1999). In this paper the vertical turbulence component $w_{T}^{*}$ acts as an external random forcing, whereas the longitudinal component of turbulence $u_{T}^{*}$ acts as a parametric random excitation.

The turbulent velocities $w_{T}^{*}$ and $u_{T}^{*}$ are obtained from the widely used, simple but realistic, Dryden model (Poirel and Price, 2003a). The basic representation of the model is given in the frequency domain. The spectral shape of the two turbulence components are different from one another; however, the overall characteristics of the system are governed by the scale of turbulence $L^{*}$ and intensity (given by the variance $D_{1}^{*}$ and $D_{2}^{*}$ ), which are common to both components

$$
S_{u_{T}^{*}}(\omega)=2 D_{2}^{*}\left(\frac{2 L^{*}}{\pi Q_{m}^{*}}\right) \frac{1}{1+\left(\frac{L^{*} \omega}{Q_{m}^{*}}\right)^{2}} \quad S_{w_{T}^{*}}(\omega)=2 D_{1}^{*}\left(\frac{L^{*}}{\pi Q_{m}^{*}}\right) \frac{1+3\left(\frac{L^{*} \omega}{Q_{m}^{*}}\right)^{2}}{\left[1+\left(\frac{L^{*} \omega}{Q_{m}^{*}}\right)^{2}\right]^{2}}
$$

The aero-elastic equations of motion are formed by combining Eq. (2.1) and Eq. (2.3), which gives a set of two integro-differential equations.

Let $\mathbf{x}=\left[x_{1}, x_{2}, x_{3}, x_{4}, x_{5}, x_{6}, x_{7}, x_{8}\right]=\left[\alpha, \dot{\alpha}, \xi, \dot{\xi}, R_{1}, \dot{R}_{1}, R_{2}, \dot{R}_{2}\right]$, Eq. (2.1) can be expressed as

$$
\begin{aligned}
& \dot{x}_{1}=x_{2} \\
& \dot{x}_{2}=a_{21} x_{1}+a_{22} x_{2}+a_{23} x_{3}+a_{24} x_{4}+a_{25} x_{5}+a_{26} x_{6}+a_{27} x_{7}+a_{28} x_{8}+a_{29} M\left(x_{1}\right) \\
& \dot{x}_{3}=x_{4} \\
& \dot{x}_{4}=a_{41} x_{1}+a_{42} x_{2}+a_{43} x_{3}+a_{44} x_{4}+a_{45} x_{5}+a_{46} x_{6}+a_{47} x_{7}+a_{48} x_{8}+a_{49} M\left(x_{1}\right) \\
& \dot{x}_{5}=x_{6} \\
& \dot{x}_{6}=a_{61} x_{1}+a_{62} x_{2}+a_{63} x_{3}+a_{64} x_{4}+a_{65} x_{5}+a_{66} x_{6}+a_{67} x_{7}+a_{68} x_{8} \\
& \dot{x}_{7}=x_{8} \\
& \dot{x}_{8}=a_{81} x_{1}+a_{82} x_{2}+a_{83} x_{3}+a_{84} x_{4}+a_{85} x_{5}+a_{86} x_{6}+a_{87} x_{7}+a_{88} x_{8}+\frac{w_{T}}{Q_{m}}
\end{aligned}
$$

where

$$
\xi=\frac{h}{b} \quad Q_{m}=\frac{Q_{m}^{*}}{b \omega_{\alpha}} \quad w_{T}=\frac{w_{T}^{*}}{b \omega_{\alpha}} \quad R_{i}=\frac{R_{i}^{*} b^{2}}{Q_{m}^{*}} \quad(i=1,2)
$$

\section{Solving the stationary probability density function by stochastic averaging and a modified averaging method}

Equations (2.5) can be expressed as $\dot{\mathbf{x}}=\mathbf{F}\left(x_{i}, Q_{m}, \tau\right)$. Let the inflow velocity $Q_{m}$ be a bifurcation parameter, and $Q_{m 0}$ the bifurcation critical point. The Jacobian matrix at $Q_{m 0}$ can be obtained for the deterministic system whose eigenvalues include a pair of pure imaginary roots and other roots with negative real parts. Let $Q_{m}=Q_{m 0}+\mu$, and $\mu$ be a perturbation near the Hopf bifurcation point. Hence, the Jacobian matrix with respect to the perturbation can be expressed as

$$
\mathbf{A}(\mu)=\mathbf{A}(0, \mu, 0)=\left.\frac{\partial \mathbf{F}}{\partial x}\right|_{\substack{x=0 \\ Q m=Q_{m 0}+\mu \\ t=0}}
$$

Expanding Eq. (3.1) at $\mu=0$ yields, $\mathbf{A}(\mu)=\mathbf{A}_{0}+\left.D \mathbf{A}(\mu)\right|_{\mu=0} \mu=\mathbf{A}_{0}+\mathbf{A}_{1} \mu$. Hence, Eqs. (2.5) can be rewritten as

$$
\dot{\mathbf{x}}=\left(\mathbf{A}_{0}+\mathbf{A}_{1} \mu\right) \mathbf{x}+\left[\mathbf{F}\left(x_{i}, Q_{m}, t\right)-\left(\mathbf{A}_{0}+\mathbf{A}_{1} \mu\right) \mathbf{x}\right]=\mathbf{A}_{0} \mathbf{x}+\mathbf{F}_{a}\left(x_{i}, \mu\right)
$$


where $\mathbf{F}_{a}\left(x_{i}, \mu\right)$ is a quadratic polynomial, and $\dot{\mathbf{x}}=\mathbf{A}_{0} \mathbf{x}$ is the derived system. Let us assume the solution of the derived system as

$$
\mathbf{x}=\mathbf{G}(\tau) \mathbf{b}
$$

where $\mathbf{G}(\tau)=\mathbf{T E}(\tau)$ is the fundamental matrix of $\mathbf{T}=\left[\varphi_{1}, \varphi_{2}, \varphi_{3}, \varphi_{4}, \varphi_{5}, \varphi_{6}, \varphi_{7}, \varphi_{8}\right]$, $\mathbf{E}(\tau)=\operatorname{diag}\left(\mathrm{e}^{\lambda_{1} \tau}, \mathrm{e}^{\lambda_{2} \tau}, \mathrm{e}^{\lambda_{3} \tau}, \mathrm{e}^{\lambda_{4} \tau}, \mathrm{e}^{\lambda_{5} \tau}, \mathrm{e}^{\lambda_{6} \tau}, \mathrm{e}^{\lambda_{7} \tau}, \mathrm{e}^{\lambda_{8} \tau}\right), \lambda_{j}$ is the eigenvalue of Jacobian matrix, $\lambda_{1}, \lambda_{2}$ is the pair of pure imaginary numbers, and the other $\operatorname{six}\left(\lambda_{3}, \ldots, \lambda_{8}\right)$ have negative real parts, $\varphi_{j}$ is the eigenvector corresponding to each eigenvalue. By applying the method of variation of constants (Hao and Wu, 2018), substituting Eq. (3.3) into Eq. (3.2), we have

$$
\dot{\mathbf{b}}=\left.\mathbf{G}^{*}(\tau) \mathbf{F}_{a}\left(x_{i}, \mu\right)\right|_{\mathbf{x}}=\mathbf{G}(\tau) \mathbf{b}=\mathbf{H}\left(b_{i}, \mu, \tau\right)=\left[H_{1}, H_{2}, H_{3}, H_{4}, H_{5}, H_{6}, H_{7}, H_{8}\right]^{\mathrm{T}}
$$

where $\mathbf{G}^{*}(\tau)$ is the inverse of the fundamental matrix $\mathbf{G}(\tau)$. In order to derive the solution in a more familiarized familiar form, we assume $\mathbf{b}$ as a function of $\tau$

$$
b_{1}=r \mathrm{e}^{\mathrm{i} \theta} \quad b_{2}=r \mathrm{e}^{-\mathrm{i} \theta} \quad b_{i}=b_{i}(\tau) \quad i=3, \ldots, 8
$$

where $r$ is the aero-elastic modal amplitude, $\theta$ is the generalized phase-angle.

Since we are only interested in long term behavior of the system when $\tau$ approaches to infinity, $b_{i}=0(i=3, \ldots, 8)$. Therefore, we obtain the normal equation by substituting Eq. (3.5) into Eq. (3.4)

$$
\begin{aligned}
& \dot{r}=\varepsilon\left(H_{1} \cos \theta+H_{2} \sin \theta\right)=\varepsilon \Phi(r, \theta, \mu) \\
& \dot{\theta}=\frac{\varepsilon\left(-H_{1} \sin \theta+H_{2} \cos \theta\right)}{r_{j}}=\varepsilon \Phi^{*}(r, \theta, \mu)
\end{aligned}
$$

where

$$
\begin{aligned}
\Phi(r & \theta, \mu)=[-2.3618 \cos (0.3644 \tau-2 \theta)-2.5026 \sin (0.3644 \tau-2 \theta) \\
& +0.0308 \cos (0.7288 \tau-4 \theta)-1.5246 \sin (0.7288 \tau-4 \theta) \\
& +0.1972 \cos (1.0932 \tau-6 \theta)-0.2137 \sin (1.0932 \tau-6 \theta)-2.2054] k_{\alpha 5} r^{5} \\
& +[-0.3695 \cos (0.3644 \tau-2 \theta)-0.5071 \sin (0.3644 \tau-2 \theta) \\
& +0.0396 \cos (0.7288 \tau-4 \theta)-0.1858 \sin (0.7288 \tau-4 \theta)-0.4324] k_{\alpha 3} r^{3} \\
& +\mu\left\{[-0.00015 \cos (0.3644 \tau-2 \theta)-0.00166 \sin (0.3644 \tau-2 \theta)-0.00097] \mu_{T}^{2}(\tau)\right. \\
& +[-0.00239 \cos (0.3644 \tau-2 \theta)-0.0111 \sin (0.3644 \tau-2 \theta)-0.00737] \mu_{T}(\tau) \\
& +[0.00064 \cos (0.3644 \tau-2 \theta)+0.01657 \sin (0.3644 \tau-2 \theta)+0.03230]\} \\
& +[0.0131 \cos (0.1822 \tau-\theta)+0.0120 \sin (0.1822 \tau-\theta)] w_{T}(\tau) \\
\Phi^{*} & r, \theta, \mu)=[-3.0071 \cos (0.3644 \tau-2 \theta)-0.5017 \sin (0.3644 \tau-2 \theta) \\
& -1.3192 \cos (0.7288 \tau-4 \theta)-0.5748 \sin (0.7288 \tau-4 \theta) \\
& -0.2137 \cos (1.0932 \tau-6 \theta)-0.1972 \sin (1.0932 \tau-6 \theta)-1.8948] k_{\alpha 5} r^{4} \\
& +[-0.3715-0.1858 \cos (0.7288 \tau-4 \theta)-0.0396 \sin (0.7288 \tau-4 \theta) \\
& -0.5731 \cos (0.3644 \tau-2 \theta)-0.0048 \sin (0.3644 \tau-2 \theta)] k_{\alpha 3} r^{2} \\
& +\mu\left\{[-0.00135+0.00015 \sin (0.3644 \tau-2 \theta)-0.00166 \cos (0.3644 \tau-2 \theta)] u_{T}^{2}(\tau)\right. \\
& \left.+[-0.00802-0.01107 \cos (0.3644 \tau-2 \theta)+0.00239 \sin (0.3644 \tau-2 \theta)] u_{T}(\tau)\right\} \\
& +\frac{1}{r}[0.01201 \cos (0.7622 \tau+\theta)-0.01307 \sin (0.7622 \tau+\theta)] w_{T}(\tau)
\end{aligned}
$$


The normal equation is in $r$ and $\theta$. By substituting $\mathbf{G}(\tau)=\mathbf{T E}(\tau)$ and $b$ into $\mathbf{x}=\mathbf{G}(\tau) \mathbf{b}$, we have an expression of $\mathbf{x}$, where $(\varphi=0.1822 \tau-\theta)$

$$
\mathbf{x}=\left[\begin{array}{c}
\alpha \\
\dot{\alpha} \\
\xi \\
\dot{\xi}
\end{array}\right]=\left[\begin{array}{c}
2.3859 r \cos \varphi+0.6544 r \sin \varphi \\
0.1192 r \cos \varphi-0.4347 r \sin \varphi \\
1.5499 r \cos \varphi-0.1270 r \sin \varphi \\
-0.0231 r \cos \varphi-0.2824 r \sin \varphi
\end{array}\right]
$$

The stochastic average and deterministic average can be utilized in Eqs. (3.6), and we obtain the following stochastic equations for the aero-elastic modal amplitude $r$ and $\theta$

$$
\begin{aligned}
d r & =\left[-2.2054 k_{\alpha 5} r^{5}-0.4324 k_{\alpha 3} r^{3}+\left(\frac{3.876 \cdot 10^{-4} \mu^{2} S_{u_{T}}\left(\omega_{1}\right)}{\pi}+0.0323 \mu\right) r\right. \\
& \left.+\frac{2.474 \cdot 10^{-4} S_{w_{T}}\left(\omega_{1}\right)}{r \pi}\right] d t \\
& +\sqrt{\frac{3.7208 \cdot 10^{-4} S_{u_{T}}\left(\omega_{1}\right)}{\pi} \mu^{2} r^{2}+\frac{4.9481 \cdot 10^{-4} S_{w_{T}}\left(\omega_{1}\right)}{\pi}} d W_{1}(t) \\
d \theta & =\left(-1.8948 k_{\alpha 5} r^{4}-0.3715 k_{\alpha 3} r^{2}-0.0028 \mu\right) d t \\
& +\sqrt{\frac{4.0375 \cdot 10^{-4} \mu^{2} S_{u_{T}}\left(\omega_{1}\right)}{\pi}+\frac{4.9481 \cdot 10^{-4} S_{w_{T}}\left(\omega_{1}\right)}{r^{2} \pi}} d W_{2}(t)
\end{aligned}
$$

where $W_{1}(t)$ and $W_{2}(t)$ are two independent Winer processes. It is worth pointing out that $\dot{r}$ does not depend on $\theta$, allowing us further to develop a probability density for $r$, rather than a joint density for $r$ and $\theta$. The probability density $p(r, \tau)$ of the instantaneous aero-elastic modal amplitude $r$ satisfies the Fokker-Planck-Kolmogorov equation (Hao and $\mathrm{Wu}, 2018$ )

$$
\begin{aligned}
& \frac{\partial p(r, \tau)}{\partial t}=-\frac{\partial}{\partial r}\left\{\left[-2.2054 k_{\alpha 5} r^{5}-0.4324 k_{\alpha 3} r^{3}\right.\right. \\
& \left.\left.\quad+\left(\frac{3.876 \cdot 10^{-4} \mu^{2} S_{u_{T}}\left(\omega_{1}\right)}{\pi}+0.0323 \mu\right) r+\frac{2.474 \cdot 10^{-4} S_{w_{T}}\left(\omega_{1}\right)}{r \pi}\right] p(r, t)\right\} \\
& \quad+\frac{1}{2} \frac{\partial^{2}}{\partial r^{2}}\left[\left(\frac{3.7208 \cdot 10^{-4} S_{u_{T}}\left(\omega_{1}\right)}{\pi} \mu^{2} r^{2}+\frac{4.9481 \cdot 10^{-4} S_{w_{T}}\left(\omega_{1}\right)}{\pi}\right) p(r, t)\right]
\end{aligned}
$$

By solving the FPK equation, we obtain an analytical solution of the system stationary probability density function

$$
p_{s}(r)=\kappa R\left(r, D_{1}, D_{2}, L, k_{\alpha 3}, k_{\alpha 5}, \mu\right) \exp \left[Q\left(r, D_{1}, D_{2}, L, k_{\alpha 3}, k_{\alpha 5}, \mu\right)\right]
$$

where $\kappa$ is the normalization coefficient, $D_{i}=D_{i}^{*} /\left(b^{2} \omega_{\alpha}^{2}\right), i=1,2, L=L^{*} / b, k_{\alpha 3}=K_{\alpha 3} / K_{\alpha}$, $k_{\alpha 5}=K_{\alpha 5} / K_{\alpha}$ and

$$
\begin{aligned}
& R\left(r, D_{1}, D_{2}, L, k_{\alpha 3}, k_{\alpha 5}, \mu\right)=r\left\{\left[3.4945 \cdot 10^{-5} \mu^{2} D_{2} L r^{2}\left(1+0.00178 L^{2}\right)\right.\right. \\
& \left.\left.\quad+2.3236 \cdot 10^{-5} D_{1} L\left(1+0.005358 L^{2}\right)\right] \frac{1}{\left(1+0.00178 L^{2}\right)^{2}}\right\}^{B\left(r, D_{1}, D_{2}, L, k_{3}, k_{5}, \mu\right)}
\end{aligned}
$$




$$
\begin{aligned}
& B\left(D_{1}, D_{2}, L, k_{\alpha 3}, k_{\alpha 5}, \mu\right)=\left\{-\frac{1}{2}\left(1+0.00178 L^{2}\right) L \mu^{6} D_{2}^{3}\right. \\
& \quad-27902.50849 k_{\alpha 5} D_{1}^{2}\left(1+0.005358 L^{2}\right)^{2} \\
& \quad+8226.934 k_{\alpha 3} D_{1} \mu^{2} D_{2}\left(1+0.005358 L^{2}\right)\left(1+0.00178 L^{2}\right) \\
& \quad+28616.38737 \mu^{4} D_{2}^{2}\left[0.03230 \mu\left(1+0.00178 L^{2}\right)\right. \\
& \left.\left.\quad+3.64017 \cdot 10^{-5} \mu D_{2} L\left(1+0.00178 L^{2}\right)^{2}\right]\right\} \frac{1}{\left(1+0.00178 L^{2}\right) D_{2}^{3} L \mu^{6}} \\
& Q\left(r, D_{1}, D_{2}, L, k_{\alpha 3}, k_{\alpha 5}, \mu\right)=\left\{-3155.69066 k_{\alpha 5} D_{2}\left(1+0.00178 L^{2}\right) \mu^{2} r^{4}\right. \\
& \quad+\left[-12372.86379 k_{\alpha 3} D_{2}\left(1+0.00178 L^{2}\right) \mu^{2}\right. \\
& \left.\left.\quad+341963.86368 k_{\alpha 5} D_{1} D_{2}\left(1+0.005358 L^{2}\right)\right] r^{2}\right\} \frac{1}{D_{2}^{2} L \mu^{4}}
\end{aligned}
$$

The relations between $\alpha, \dot{\alpha}, \xi, \dot{\xi}$ and $r, \theta$ are defined in Eq. (3.7). Thus, we have analytical expressions of $p_{s}\left(r_{\alpha}\right)$ and $p_{s}\left(r_{h}\right)$, which are PDFs (probability density functions) of the pitch angle and plunge displacement

$$
\begin{aligned}
& p_{s}\left(r_{\alpha}\right)=\kappa R_{1}\left(r_{\alpha}, D_{1}, D_{2}, L, k_{\alpha 3}, k_{\alpha 5}, \mu\right) \exp \left[Q_{1}\left(r_{\alpha}, D_{1}, D_{2}, L, k_{\alpha 3}, k_{\alpha 5}, \mu\right)\right] \\
& p_{s}\left(r_{\xi}\right)=\kappa R_{2}\left(r_{\xi}, D_{1}, D_{2}, L, k_{\alpha 3}, k_{\alpha 5}, \mu\right) \exp \left[Q_{2}\left(r_{\xi}, D_{1}, D_{2}, L, k_{\alpha 3}, k_{\alpha 5}, \mu\right)\right]
\end{aligned}
$$

where

$$
\begin{aligned}
& Q_{1}\left(r_{\alpha}, D_{1}, D_{2}, L, k_{\alpha 3}, k_{\alpha 5}, \mu\right)=\left\{-84.2281 k_{\alpha 5} D_{2}\left(1+0.00178 L^{2}\right) \mu^{2} r_{\alpha}^{4}\right. \\
& \quad+\left[-2021.3966 k_{\alpha 3} D_{2}\left(1+0.00178 L^{2}\right) \mu^{2}\right. \\
& \left.\left.\quad+55867.79304 k_{\alpha 5} D_{1}\left(1+0.005358 L^{2}\right)\right] r_{\alpha}^{2}\right\} \frac{1}{D_{2}^{2} L \mu^{4}} \\
& \quad R_{1}\left(r_{\alpha}, D_{1}, D_{2}, L, k_{\alpha 3}, k_{\alpha 5}, \mu\right)=r_{\alpha}\left\{\left[2.3076 \cdot 10^{-6} \mu^{2} D_{2} L r_{\alpha}^{2}\left(1+0.00178 L^{2}\right)\right.\right. \\
& \left.\left.\quad+9.3919 \cdot 10^{-6} D_{1} L\left(1+0.005358 L^{2}\right)\right] \frac{1}{\left(1+0.00178 L^{2}\right)^{2}}\right\}^{B\left(D_{1}, D_{2}, L, k_{3}, k_{5}, \mu\right)} \\
& \quad Q_{2}\left(r_{\xi}, D_{1}, D_{2}, L, k_{\alpha 3}, k_{\alpha 5}, \mu\right)=\left\{-539.5858 k_{\alpha 5} D_{2}\left(1+0.00178 L^{2}\right) \mu^{2} r_{\xi}^{4}\right. \\
& \quad+\left[-5116.2679 k_{\alpha 3} D_{2}\left(1+0.00178 L^{2}\right) \mu^{2}\right. \\
& \left.\left.\quad+1.41405 \cdot 10^{5} k_{\alpha 5} D_{1}\left(1+0.005358 L^{2}\right)\right] r_{\xi}^{2}\right\} \frac{1}{D_{2}^{2} L \mu^{4}} \\
& R_{2}\left(r_{\xi}, D_{1}, D_{2}, L, k_{\alpha 3}, k_{\alpha 5}, \mu\right)=r_{\xi}\left\{\left[9.2921 \cdot 10^{-6} \mu^{2} D_{2} L r_{\xi}^{2}\left(1+0.00178 L^{2}\right)\right.\right. \\
& \left.\left.\quad+1.4942 \cdot 10^{-5} D_{1} L\left(1+0.005358 L^{2}\right)\right] \frac{1}{\left(1+0.00178 L^{2}\right)^{2}}\right\}^{B\left(D_{1}, D_{2}, L, k_{3}, k_{5}, \mu\right)}
\end{aligned}
$$

\section{Bifurcation analysis of the deterministic system}

In a non-dimensional form, the airfoil parameters used in the analysis, are taken from (Poirel and Price, 2007), and $k_{\alpha 3}=-0.3736, k_{\alpha 5}=0.2197$. The bifurcation of the deterministic system is shown in Fig. 1. The horizontal axis represents the perturbation of $Q_{m}$ in the vicinity of the bifurcation point and the vertical axis represents the aero-elastic modal amplitude $r$. The dotted lines are unstable limit cycles while the solid line is the equilibrium and stable limit cycle. Figure 1 demonstrates that the periodic solution produced by Hopf bifurcation has involved a second bifurcation at $\mu=-0.417$. The critical velocity of saddle node bifurcation points is advanced. The bifurcation diagram shows discontinuous and unstable limit cycles, where the 
system appears to be multi-stable. The figure shows that the results solved from the first order approximation via, respectively, the modified averaging method and the averaging method. Blue stars shown in Fig. 1 are the numerical results by adopting the Rung-Kutta 4th order algorithm. It is obvious that the numerical approaches nearly over-write the analytical methods (black line), which validates the proposed solving approach. The modified averaging method gives accurate results by making a dimension reduction process.

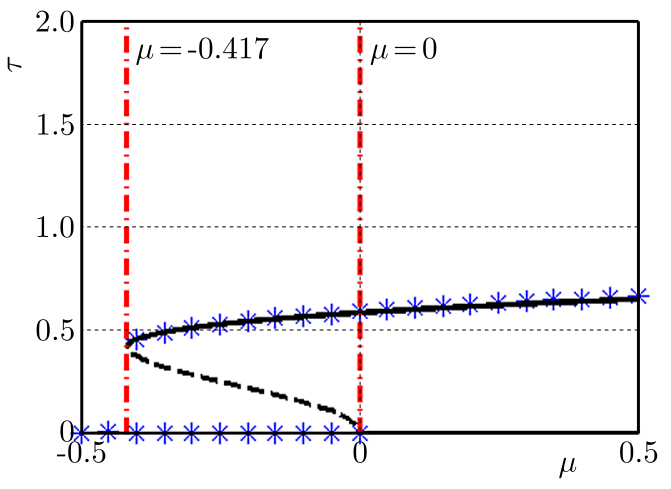

Fig. 1. Bifurcation diagram of the deterministic system

In Fig. $1\left(Q_{m}=Q_{m 0}+\mu\right), \mu=0$ corresponds to $Q_{H}=4.3154$, where $Q_{H}=4.3154$ is the Hopf bifurcation point, which is known as the first critical speed or the linear critical flow speed. The nonlinear criticality arrives when $\mu=-0.417$, which corresponds to $Q_{A}=3.8983$, where the second bifurcation (saddle-node bifurcation) occurs. Nevertheless, the nonlinear critical velocity of the system for a large amplitude vibration LCO is smaller than the nonlinear critical velocity.

\section{Application of the stochastic singularity theory and analysis of the parameter influence}

For this non-linear airfoil, we can analyze the system stationary response behavior by investigating the standard equations of aeroelastic modality amplitude $r$ and angle $\theta$. First, the bifurcation equation of the $2 \mathrm{DOF}$ airfoil model is selected as

$$
g(r)=R\left(r, D_{1}, D_{2}, L, k_{\alpha 3}, k_{\alpha 5}, \mu\right) \exp \left[Q\left(r, D_{1}, D_{2}, L, k_{\alpha 3}, k_{\alpha 5}, \mu\right)\right]-p_{s}(r)=0
$$

The transition set $H$ (hysteresis set) and $D L$ (double-limit point set) of the non-linear flutter system corresponding to bifurcation equation (5.1) is solved by using the singularity theory. Types of transfer sets and their calculation methods are the same as those in (Hao and Wu, 2013). Here, the aero-elastic modality amplitude $r$ is a state variable; the stationary probability density $p_{s}(r)$ is a bifurcation parameter; the perturbation $\mu$ of vertical and longitudinal turbulent intensities $D_{1}$ and $D_{2}$ of the longitudinal inflow velocity $Q_{m}$ near the Hopf bifurcation point is the unfolding parameter.

For $L=50$, Fig. 2 shows the transition set profile in the $\left(D_{1}, \mu\right)$ plane for several $D_{2}$. Here, the dotted line is the hysteresis set $H$, and the dot-and-dash line is a double limit point set $D L$. The hysteresis set $H$ and $D_{1}=D_{2}=0$ correspond to the linear and nonlinear critical flow speed $\left(Q_{H}=4.3154, Q_{A}=3.8983\right)$ in Fig. 2. The transition set $H$ and $D L$ in the $\left(D_{1}, \mu\right)$ in the parameter plane divide the parameter plane into different parameter domains marked as $1 \mathrm{a}, 2$, 3 and $1 b$.

Figure 3 shows the stationary PDF of the aero-elastic modal amplitude $r$ found at points selected in the parameter domains divided by the transition set curve. Here, the black curve is the theoretical result, and the blue asterisked line is the result of Monte Carlo simulation. The 


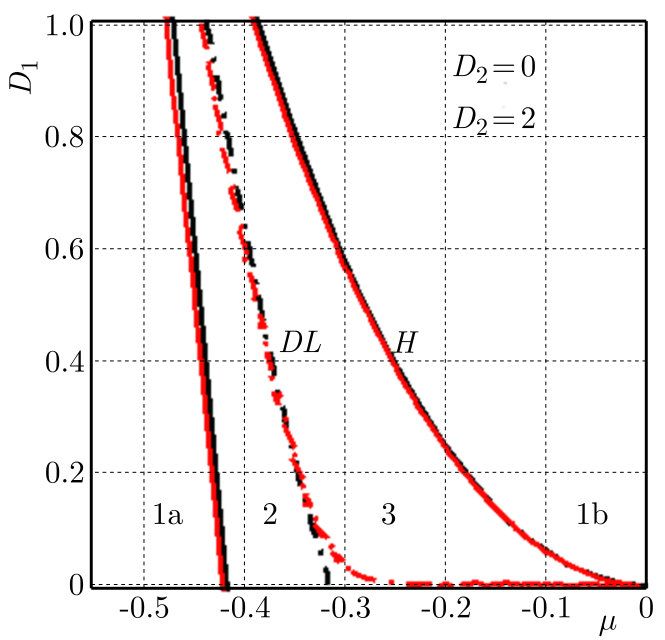

Fig. 2. Critical conditions of P-bifurcation (transition sets) for two intensities of longitudinal turbulence

Rung-Kutta 4-th order algorithm has been adopted to acquire the time history of each sample, so that after 10000 iterations the simulation could run into a steady state. We ran the Monte Carlo simulation with a sample number set of $10^{6}$. The two results agree well with each other suggesting that our theoretical calculation is reliable. Also the time responses of $\alpha$ and $\dot{\alpha}$ are shown in Fig. 3 corresponding to the same parameters.

As the perturbation $\mu$ to the longitudinal inflow velocity continues to increase, in the transition set profile (Fig. 2) the system undergoes three stochastic P-bifurcations. When $\mu$ increases to 0 , the longitudinal inflow velocity reaches the system linear critical velocity $Q_{H}$. In this case, there will be only sharp airfoil flutter, and variation of the turbulent excitation intensity will not cause any qualitative variation to the system stationary response.

The PDF of the aero-elastic modal amplitude $r$ obtained from region 1 (in Fig. 2) of the $\left(D_{1}, \mu\right)$ parameter plane is shown in Fig. 3a(1a). No large amplitude vibration is observed in this region, and the time responses of $\alpha$ and $\dot{\alpha}$ shown in Fig. 3d(1b) also verify the probabilistic distribution.

Another peak occurs in the PDF curve when the parameter set passes through region 1a to region 2 (in Fig. 2) of the $\left(D_{1}, \mu\right)$ plane, as shown in Fig. 3b(2). The stochastic P-bifurcation has happened and a large amplitude vibration can be found, but small vibration still exists. Time domain results of $\alpha, \dot{\alpha}$ are shown in Fig. 3b(right).

When the parameter set passes through region 2 to region 3 (in Fig. 2) of the $\left(D_{1}, \mu\right)$ plane, the second peak of the PDF curve begins to be higher than the first peak. This means that region 3 corresponds mainly to the large amplitude vibration and the duration of small amplitude vibration becomes short, Fig. 3c(right).

Further increase in the airspeed $Q_{m}$ causes the third stochastic P-bifurcation to occur, and the PDF curves reduce to only one peak. The large amplitude vibration dominates the dynamics in this case, as shown in Fig. 3d(1b) and Fig. 3d(right), and the system behaves like a diffused limit cycle.

Comparing the two groups of transition set profiles presented above, we can find that as the longitudinal turbulent intensity $D_{2}$ increases, the transition set curve moves in the direction where $\mu\left(Q_{m}=Q_{m 0}+\mu\right)$ decreases. In the $\left(D_{1}, \mu\right)$ parameter plane, as $D_{2}$ continues to increase, parameter domain $1 \mathrm{~b}$ also continues to enlarge. When the parameters are selected within transition set parameter domain $1 \mathrm{~b}$, the airfoil continues to undergo sharp stochastic flutter. An increase in the longitudinal turbulent intensity $D_{2}$ increases the parameter range for sharp airfoil flutter, thus compromising the system stationary response. 
(a)

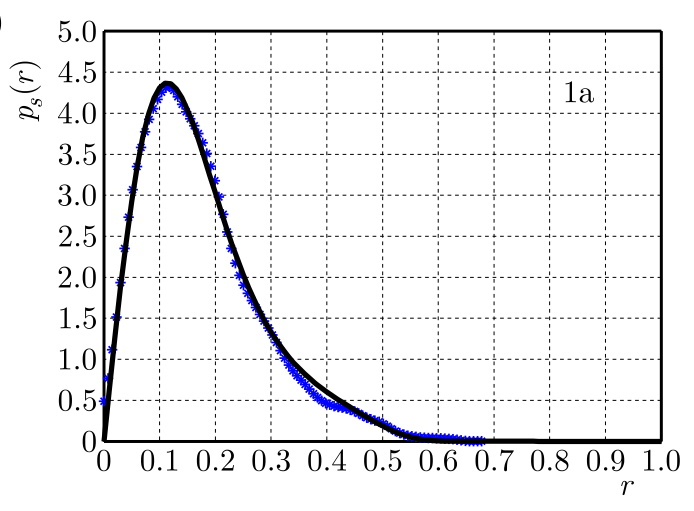

(b)

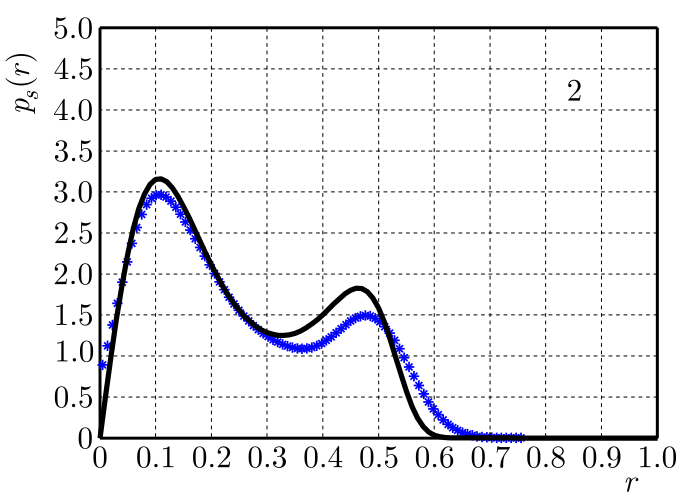

(c)

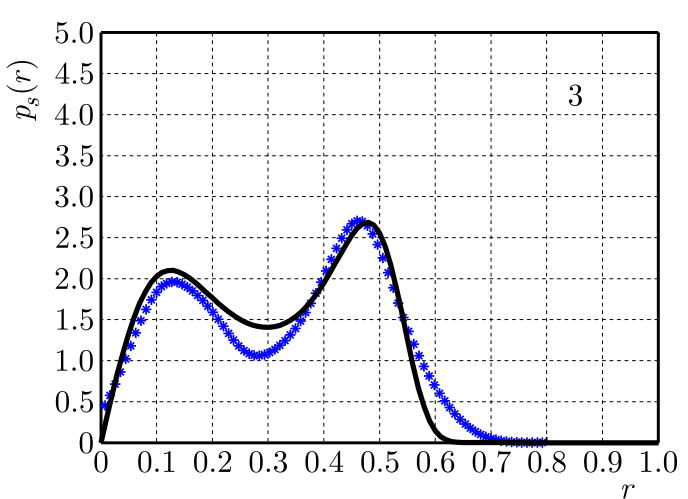

(d)

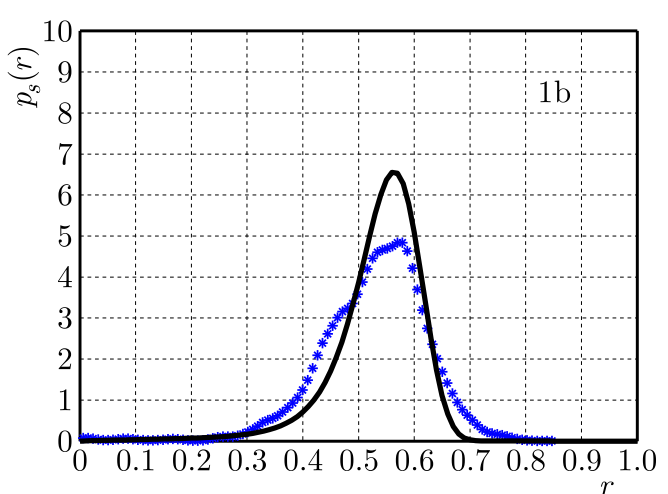

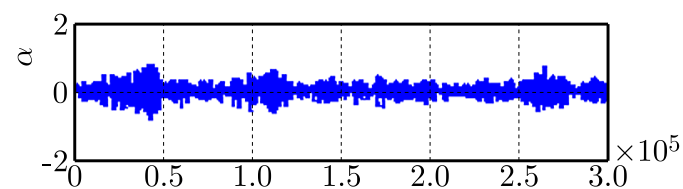
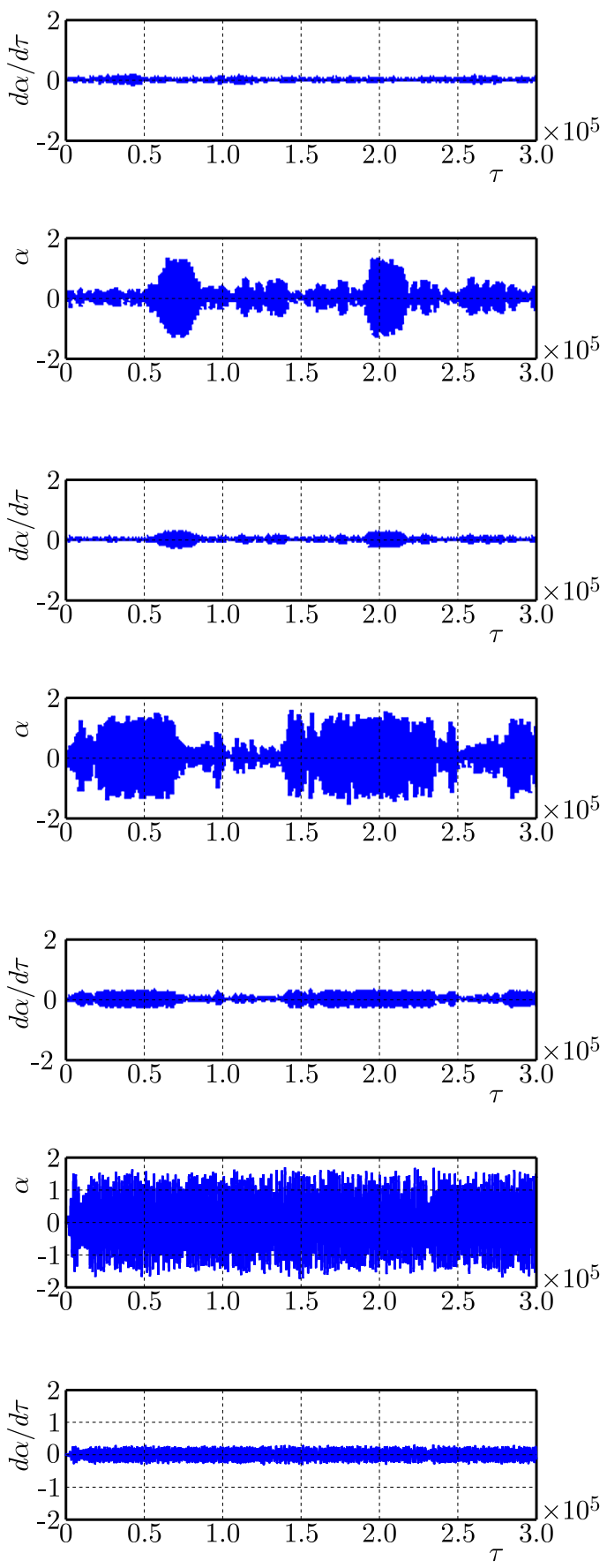

Fig. 3. PDF profile (bifurcation diagram) of $r$ and time history of $\alpha$, $\dot{\alpha}$ : (a) $\mu=-0.6, D_{1}=0.8$, (b) $\mu=-0.4, D_{1}=0.45$, (c) $\mu=-0.38, D_{1}=0.55$, (d) $\mu=-0.1, D_{1}=1$ 
With the increase of $D_{1}$, the value $\mu$ of the parameter set corresponding to the left hysteresis set $H$ gradually decreases (Fig. 2). The crossing is the occurrence of stochastic P-bifurcation, and the large amplitude vibration is observed at region 2 , so it means that the critical velocity for sharp airfoil flutter will gradually decrease too. Similar to what happens when the longitudinal turbulent intensity increases, the critical velocity for sharp stochastic airfoil flutter comes earlier. An increase in the longitudinal and vertical turbulent velocities $D_{2}$ and $D_{1}$ will both cause the critical velocity for sharp stochastic airfoil flutter to come earlier.

Figure 4 shows the effects of the turblent scale $L$ on the linear and nonlinear critical velocities $Q_{T H}$ and $Q_{T A}$ under turbulent perturbation when the vertical and longitudinal turbulent velocities are constant. As the turbulent scale $L$ increases, both $Q_{T H}$ and $Q_{T A}$ become smaller, implying that an increase in the turbulent scale will cause the critical velocity for sharp stochastic airfoil flutter to occur earlier.

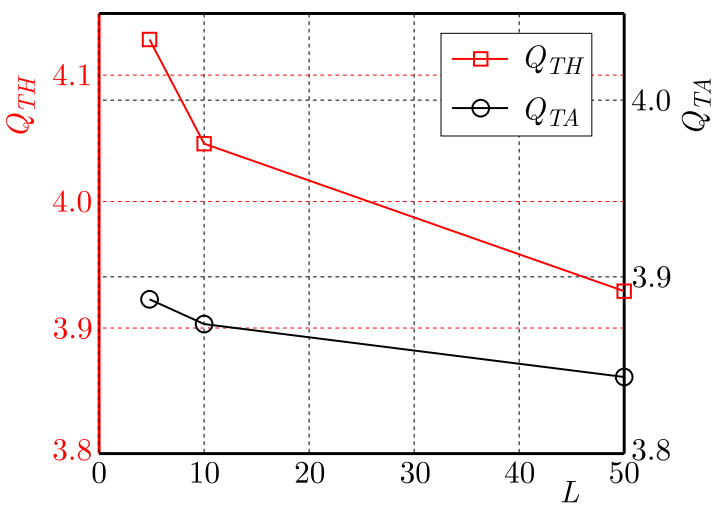

Fig. 4. Influence of $L$ on velocity when $D_{1}=D_{2}=1$
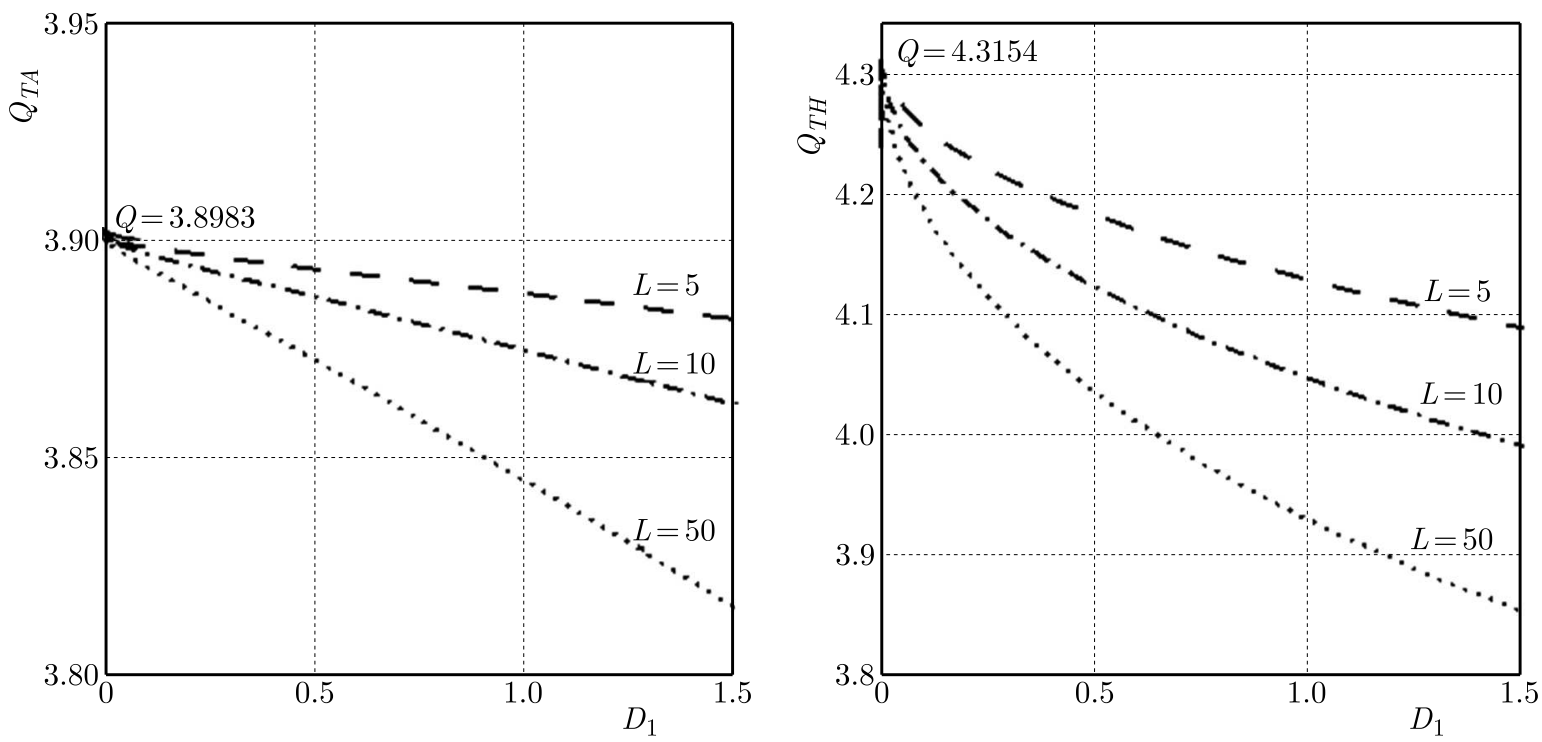

Fig. 5. Variation of the critical velocity

For $D_{1} \neq 0, D_{2}=1$, Fig. 5 shows how the critical velocities $Q_{T H}$ and $Q_{T A}$ vary with $D_{1}$ by different terms of turbulent scales $L$. When $L=5$, the turbulent scale is small, and both linear and nonlinear critical velocities $Q_{T H}$ and $Q_{T A}$ decrease slowly with the increase of vertical turbulent intensity. Comparing the different curves in this chart, we will see that an increase in $L$ accelerates the $Q_{T H}$ and decreases the $Q_{T A}$. At the same time the other parameters are constant, whereas as increase in the turbulent scale decreases the critical velocity for sharp stochastic airfoil flutter. An increase in both the turbulent scale $L$ and vertical and longitudinal 
turbulent intensities $D_{1}$ and $D_{2}$ can makes the critical velocity for sharp stochastic airfoil flutter come earlier.

\section{Conclusions}

The stochastic flutter and stochastic P-bifurcation of an airfoil in a two-dimensional turbulent flow are examined. By addressing the potential effects of two-dimensional turbulence, the binary airfoil model is expanded to eight dimensions, taking into account five-fold structural nonlinearity and complexity of the aerodynamic model. A combination of the modified averaging method with the stochastic averaging method reduces the difficulty of applying the stochastic averaging method in high-dimensional systems, and is successfully applied to solve this high-dimensional system.

Analysis of the stochastic singularity and discussion of the system stochastic P-bifurcation discovered that in the linearly stable area with the presence of a two-dimensional flow, an increase in the turbulent intensity leads to a reduction of the critical flutter velocity for sharp stochastic airfoil flutter, and makes the time for sharp airfoil flutter come earlier. Moreover, when other parameters are constant, the increase in the turbulent scale brings forward the critical velocity for sharp stochastic flutter, and it accelerates the decreasing rate of the critical velocity.

\section{Appendix}

$$
\begin{aligned}
& f_{1}=\frac{1}{a_{1} c_{2}-a_{2} c_{1}} \quad f_{2}=\frac{1}{a_{2} c_{1}-a_{1} c_{2}} \\
& a_{21}=f_{1}\left(a_{2} c_{5}-a_{5} c_{2}\right) \quad a_{22}=f_{1}\left(a_{2} c_{3}-a_{3} c_{2}\right) \quad a_{23}=f_{1}\left(a_{2} c_{6}-a_{6} c_{2}\right) \\
& a_{24}=f_{1}\left(a_{2} c_{4}-a_{4} c_{2}\right) \quad a_{25}=f_{1}\left(a_{2} c_{9}-a_{9} c_{2}\right) \quad a_{26}=f_{1}\left(a_{2} c_{7}-a_{7} c_{2}\right) \\
& a_{27}=f_{1}\left(a_{2} c_{10}-a_{10} c_{2}\right) \quad a_{28}=f_{1}\left(a_{2} c_{8}-a_{8} c_{2}\right) \quad a_{29}=-f_{1} c_{2} \\
& a_{41}=f_{2}\left(a_{1} c_{5}-a_{5} c_{1}\right) \quad a_{42}=f_{2}\left(a_{1} c_{3}-a_{3} c_{1}\right) \quad a_{43}=f_{2}\left(a_{1} c_{6}-a_{6} c_{1}\right) \\
& a_{44}=f_{2}\left(a_{1} c_{4}-a_{4} c_{1}\right) \quad a_{45}=f_{2}\left(a_{1} c_{9}-a_{9} c_{1}\right) \quad a_{46}=f_{2}\left(a_{1} c_{7}-a_{7} c_{1}\right) \\
& a_{47}=f_{2}\left(a_{1} c_{10}-a_{10} c_{1}\right) \quad a_{48}=f_{2}\left(a_{1} c_{8}-a_{8} c_{1}\right) \quad a_{49}=-f_{2} c_{1} \\
& a_{61}=-\frac{d_{5}}{d_{7}} \quad a_{62}=-\frac{d_{3}}{d_{7}} \quad a_{63}=-\frac{d_{6}}{d_{7}} \quad a_{64}=-\frac{d_{4}}{d_{7}} \\
& a_{65}=-\frac{d_{9}}{d_{7}} \quad a_{66}=-\frac{d_{8}}{d_{7}} \quad a_{61}=0 \quad a_{61}=0 \\
& a_{81}=a_{82}=a_{83}=a_{84}=a_{85}=a_{86}=0 \quad a_{87}=-\frac{e_{9}}{e_{7}} \quad a_{88}=-\frac{e_{8}}{e_{7}} \\
& a_{1}=1+\frac{a_{h}^{2}+\frac{1}{8}}{u r_{\alpha}^{2}} \quad a_{2}=\frac{x_{\alpha}}{r_{\alpha}^{2}} \quad a_{3}=\frac{2 \zeta_{\alpha}}{Q_{m}}+\frac{\frac{1}{2}-a_{h}}{u r_{\alpha}^{2}} U_{1}-\frac{2\left(a_{h}+\frac{1}{2}\right)\left(\frac{1}{2}-a_{h}\right)}{u r_{\alpha}^{2}} U_{1} \varphi(0) \\
& a_{4}=-\frac{2\left(a_{h}+\frac{1}{2}\right)}{u r_{\alpha}^{2}} U_{1} \varphi(0) \quad a_{5}=\frac{1}{Q_{m}^{2}}-\frac{2\left(a_{h}+\frac{1}{2}\right)}{u r_{\alpha}^{2}} U_{2} \varphi(0) \quad a_{6}=0 \\
& a_{7}=-\frac{2\left(a_{h}+\frac{1}{2}\right)\left(A_{1} b_{1}+A_{2} b_{2}\right)}{u r_{\alpha}^{2}} U_{1} \quad a_{8}=-\frac{2\left(a_{h}+\frac{1}{2}\right)\left(A_{3} b_{3}+A_{4} b_{4}\right)}{u r_{\alpha}^{2}} \\
& a_{9}=-\frac{\left(a_{h}+\frac{1}{2}\right) b_{1} b_{2}}{u r_{\alpha}^{2}} U_{1} \quad a_{10}=-\frac{2\left(a_{h}+\frac{1}{2}\right) b_{3} b_{4}}{u r_{\alpha}^{2}}
\end{aligned}
$$




$$
\begin{aligned}
& c_{1}=x_{\alpha}-\frac{a_{h}}{u} \quad c_{2}=1+\frac{1}{u} \quad c_{3}=\frac{U_{1}}{u}+\frac{2\left(\frac{1}{2}-a_{h}\right)}{\mu} U_{1} \varphi(0) \\
& c_{4}=\frac{2 \zeta_{h} \bar{\omega}}{Q_{m}}+\frac{2}{u} U_{1} \varphi(0) \quad c_{5}=\frac{2}{u} U_{2} \varphi(0) \quad c_{6}=\frac{\bar{\omega}^{2}}{Q_{m}^{2}} \quad c_{7}=\frac{2\left(A_{1} b_{1}+A_{2} b_{2}\right)}{u} U_{1} \\
& c_{8}=\frac{2\left(A_{3} b_{3}+A_{4} b_{4}\right)}{u} \quad c_{9}=\frac{b_{1} b_{2}}{u} U_{1} \quad c_{10}=\frac{2 b_{3} b_{4}}{u} \\
& d_{2}=0 \quad d_{3}=-\left(\frac{1}{2}-a_{h}\right) \quad d_{4}=-1 \\
& d_{1}=0 \quad d_{8}=b_{1}+b_{2} \quad d_{9}=b_{1} b_{2} \\
& d_{6}=0 \quad e_{7}=1 \\
& e_{i}=0 \quad(i=1, \ldots, 6) \quad b_{1}+b_{4} \\
& Q=Q_{m}+u_{T}(\tau) \quad e_{1}=b_{3} b_{4} \\
& U_{2}=\left(\frac{Q(\tau)}{Q_{m}}\right)^{2}=\left(1+\frac{u_{T}(\tau)}{Q_{m}}\right)^{2}=1+\frac{2 u_{T}(\tau)}{Q_{m}}+\left(\frac{u_{T}(\tau)}{Q_{m}}\right)^{2} \\
& \varphi(0)=1-A_{1}-A_{2} \quad \psi(0)=1-A_{3}-A_{4}
\end{aligned}
$$

\section{Acknowledgements}

This research was supported by the following projects: National Natural Science Foundation of China (No. 11902287 and No. 11172198); Science and Technology research Project of Colleges and universities in Hebei province, China (No. 2016045); The Doctoral Starting up Foundation of Yanshan University, China (B938); Science and Technology of Qinhuangdao, China (No. 2015A248).

\section{References}

1. Chassaing J.C., Lucor D., TrÉgon J., 2012, Stochastic nonlinear aeroelastic analysis of a supersonic lifting surface using an adaptive spectual method, Journal of Sound and Vibration, 331, 2, 394-411

2. Hao Y. Wu Z.-Q., 2013, Stochastic P-bifurcation of tri-stable van der Pol-Duffing osciliator, Acta Mechanica Sinica, 45, 2, 257-265

3. Haо Y., Wu Z.-Q., 2018, Random flutter of multi-stable airfoils excited parametrically in steady flows, Journal of Mechanics, 35, 3, 419-426

4. HuAng Y., FAng C., LiU X., 2010, On stochastic dynamical behaviors of binary airfoil with nonlinear structure, Acta Aeronautica et Astronautica Sinica, 31, 10, 1946-1952

5. LeE B.H.K., Price S.J., Wong Y.S., 1999, Nonlinear aeroelastic analysis of airfoils: bifurcation and chaos, Progress in Aerospace Sciences, 35, 3, 205-334

6. Poirel D.C., Dunn S., Porter J., 2005, Flutter-margin method accounting for modal parameters uncertainties, Journal of Aircraft, 42, 5, 1236-1243

7. Poirel D.C., Harris Y., AzÉmi B., 2006, Aeroelastic dynamics of a NACA 0012 airfoil in the transitional Reynolds number regime, Asme Pressure Vessels and Piping/icpvt-11 Conference

8. Poirel D.C., Harris Y., Benaissa A., 2008, Self-sustained aeroelastic oscillations of a NACA0012 airfoil at low-to-moderate Reynolds numbers, Journal of Fluids and Structures, 24, $5,700-719$

9. Poirel D.C., Mendes F., 2014, Experimental small-amplitude self-sustained pitch-heave oscillations at transitional Reynolds numbers, AIAA Journal, 52, 8, 1581-1590

10. Poirel D.C., Price S.J., 1997, Post-instability behavior of a structurally nonlinear airfoil in longitudinal turbulence, Journal of Aircraft, 34, 5, 619-627 
11. Poirel D.C., Price S.J., 2001, Structurally nonlinear fluttering airfoil in turbulent flow, $A I A A$ Journal, 39, 10, 1960-1968

12. Poirel D.C., Price S.J., 2003a, Random binary (coalescence) flutter of a two-dimensional linear airfoil, Journal of Fluids and Structures, 18, 1, 23-42

13. Poirel D.C., Price S.J., 2003b, Response probability structure of a structurally nonlinear fluttering airfoil in turbulent flow, Probabilistic Engineering Mechanics, 18, 2, 185-202

14. Poirel D.C., Price S.J., 2007, Bifurcation characteristics of a two-dimensional structurally non-linear airfoil in turbulent flow, Nonlinear Dynamics, 48, 4, 423-435

15. Poirel D.C., Yuan W., 2010, Aerodynamics of laminar separation flutter at a transitional Reynolds number, Journal of Fluids and Structures, 26, 7-8, 1174-1194

16. Price S.J., Alighanbari H., Lee B.H.K., 2013, The aeroelastic response of a two-dimensional airfoil with bilinear and cubic structural nonlinearities, Journal of Fluids and Structures, 9, 2, $175-193$

17. Yuan W., Poirel D.C., Wang B., 2013, Simulations of pitch-heave limit-cycle oscillations at a transitional Reynolds number, AIAA Journal, 51, 7, 1716-1732

18. Zhao D.M., Zhang Q.C., TAN Y., 2009, Random flutter of a 2-DOF nonlinear airfoil in pitch and plunge with freeplay in pitch, Nonlinear Dynamics, 58, 4, 643-654

19. Zheng G.Y., 2007, Complicated Responses of Supersonic Flutter System with Structural Nonlinearities, Southwest Jiaotong University

Manuscript received March 11, 2019; accepted for print August 20, 2019 